\section{Variação temporal no excesso de peso é obesidade em adolescentes de escola privada do Sul do Brasil}

\author{
Temporal variation in overweight and \\ obesity in adolescents from a private \\ school of Southern Brazil
}

\author{
Mariana Otero Xavier ${ }^{1}$ \\ Renata Moraes Bielemann² \\ Francine Villela Maciel ${ }^{1}$ \\ Marilda Borges Neutzling ${ }^{3}$ \\ Denise Petrucci Gigante ${ }^{1,2}$
}

\section{RESUMO}

O objetivo do estudo foi avaliar a variação temporal em doze anos nas prevalências de excesso de peso e obesidade em adolescentes de uma escola privada de Pelotas-RS e a associação transversal com atividade física e tempo de televisão. Foram realizados dois inquéritos transversais com estudantes do ensino médio da escola em 2000 e 2012. Foram aferidos peso e altura dos adolescentes. Valores de escore-z de IMC para idade superiores a +1 e +2 , respectivamente, classificaram o excesso de peso e obesidade. Foram considerados ativos os adolescentes que atingiram pelo menos 300 minutos/semana de atividade física em 2012 . Tempo igual ou superior a duas horas assistindo televisão foi considerado alto. Em 2000 a prevalência de excesso de peso foi de 21,5\%, enquanto que em 2012 aumentou para 30,7\% $(\mathrm{p}=0,003)$. Quanto à obesidade, a prevalência quase dobrou, passando de 5,0\% para 9,7\% $(\mathrm{p}=0,01)$. Em ambos os períodos, os dois desfechos mostraram-se mais prevalentes no sexo masculino. A prevalência de excesso de peso aumentou significativamente nos meninos $(\mathrm{p}=0,002)$, enquanto a obesidade aumentou mais nas meninas $(\mathrm{p}=0,01)$. Maiores incrementos de ambos os desfechos se deram na faixa etária dos 16 anos ou mais. A prática de atividade física não foi associada com as prevalências dos desfechos em 2012. Entretanto, assistir televisão por pelo menos duas horas/dia foi associada com incremento superior a 10 pontos percentuais na prevalência de excesso de peso. Os resultados mostraram um grande aumento nas prevalências de excesso de peso e obesidade dos adolescentes desta escola.

\section{PALAVRAS-CHAVE}

Excesso de peso; Obesidade; Estudo comparativo; Adolescente; Atividade Motora; Estilo de vida sedentário.

The aim of this study was to evaluate the temporal variation in a 12-year period of the overweight and obesity prevalence in adolescents at a private school from Pelotas-RS and the cross-sectional association with physical activity and $T V$-viewing. Two cross-sectional studies were carried-out with high school students in 2000 and 2012. Standing height and weight were measured. Overweight and obesity were defined when the $z$ scores of BMI for age were higher than +1 and +2 , respectively. Adolescents who reached at least 300 minutes/week of physical activity were considered as active in 2012. TV-viewing $\geq 2$ hours/day was considered as high. In 2000, the overweight prevalence was $21.5 \%$, whereas in 2012 increased to $30.7 \%$ (p=0.003). Concerning the obesity, the prevalence almost doubled, changing from $5.0 \%$ to $9.7 \%(p=0.001)$. In the two periods, both overweight and obesity were more prevalent in boys. Between 2000 and 2012, the overweight prevalence increased more in boys ( $p=0.002)$, whereas the obesity increased more in girls $(p=0.01)$. Both outcomes increased mainly in adolescents aged 16 years old or more. Physical activity was not associated with both outcomes in 2012. However, TV-viewing $\geq 2$ bours/day was associated with more than 10 percentage points in the overweight prevalence. The results showed a large increase in the overweight and obesity in adolescents from this school.
}

\section{KEYWORDS}

Overweight; Obesity; Comparative study; Adolescent; Motor activity; Sedentary lifestyle.
Rev Bras Ativ Fis Saúde p. 74-85 DOI:

http://dx.doi.org/10.12820/rbafs.v.19n1p74

1 Faculdade de Nutrição. Universidade Federal de Pelotas - Pelotas, RS, Brasil

2 Programa de Pós-Graduação em Epidemiologia. Universidade Federal de Pelotas - Pelotas, RS, Brasil

3 Programa de Pós-Graduação em Epidemiologia. Universidade Federal do Rio grande do Sul - Porto Alegre, RS, Brasil 


\section{INTRODUCCÃO}

A redução nas prevalências dos déficits nutricionais e a ascensão nas prevalências de excesso de peso constituem características da transição nutricional, a qual ocorre de maneira distinta conforme o nível de desenvolvimento de cada país ${ }^{1}$. No Brasil, mesmo com a coexistência de ambos os desfechos, observase declínio contínuo nas prevalências de déficits nutricionais e aumento nas prevalências excesso de peso e obesidade ${ }^{2}$.

O período da adolescência é caracterizado por intensas transformações, grande crescimento físico e desenvolvimento, com alterações fisiológicas complexas nas quais a nutrição adequada desempenha papel fundamental ${ }^{3}$. No entanto, resultados da Pesquisa Nacional de Saúde do Escolar (PeNSE), que avaliou o estado nutricional de estudantes do $9^{\circ}$ ano do ensino fundamental de escolas públicas e privadas de todas as capitais brasileiras, revelaram que o excesso de peso atingia quase um quarto da população estudada $(23,0 \%)$, sendo mais observado entre estudantes de escolas privadas ${ }^{4}$, fato que também foi constatado em estudo de revisão sobre obesidade em adolescentes brasileiros ${ }^{5}$. Ainda, a comparação de dois estudos nacionalmente representativos mostra que em trinta e quatro anos a prevalência de excesso de peso aumentou em seis vezes no sexo masculino $(3,7 \%$ vs. $21,7 \%)$ e em quase três vezes no sexo feminino $(7,6 \%$ vs. $19,4 \%)$, enquanto que a obesidade passou de $0,4 \%$ para $5,9 \%$ no sexo masculino e de $0,7 \%$ para $4,0 \%$ no sexo feminino 6 .

A tendência de aumento do excesso de peso e, em especial, da obesidade, é extremamente preocupante em virtude do maior risco que crianças e adolescentes obesos apresentam de continuarem obesos na vida adulta e virem a desenvolver comorbidades relacionadas à doença ${ }^{7}$. Ainda, evidências sugerem que complicações como a síndrome metabólica afetam especialmente crianças e adolescentes com excesso de peso ou obesidade ${ }^{8}$.

Dentre os principais fatores de risco associados ao excesso de peso estão a inatividade física e o comportamento sedentário. Dados também da PeNSE apontaram que apenas $43,1 \%$ dos adolescentes entrevistados atingiam a recomendação atual de prática de atividade física de 300 minutos/semana, não sendo encontrada nenhuma associação com o tipo de escola. Em contrapartida, o mesmo estudo encontrou que quase $80 \%$ dos estudantes assistia televisão por tempo igual ou superior a duas horas/dia, sendo a maior proporção entre estudantes da rede privada de ensino ${ }^{9}$. Paralelamente ao aumento do excesso de peso, estudos apontam uma tendência de diminuição na prática de atividades físicas por adolescentes ${ }^{10}$, ainda que em estudo realizado em Pelotas com adolescentes de 10 a 19 anos tenha sido observada estabilização nos níveis de atividade física entre 2005 e 2012, porém com declínio significativo no que se refere ao deslocamento ativo para a escola ${ }^{11}$.

O monitoramento da situação nutricional de populações jovens que permita comparações através do tempo e o reconhecimento de fatores possivelmente associados é extremamente útil para a promoção de estratégias de prevenção e controle. Sendo assim, o objetivo deste estudo foi avaliar a variação temporal em doze anos nas prevalências de excesso de peso e obesidade em adolescentes de uma escola privada de Pelotas-RS e a associação transversal com a prática de atividade física e o tempo assistindo televisão. 


\section{METODOLOGIA}

Foi realizada uma análise de variação temporal a partir de dados oriundos de dois inquéritos transversais conduzidos em uma escola privada do município de Pelotas/RS, em dois diferentes períodos de tempo (anos 2000 e 2012). Os dados coletados nesta escola no ano 2000 fizeram parte de um projeto que avaliou os fatores de risco para o excesso de peso entre adolescentes de todas as escolas privadas existentes em Pelotas na época ${ }^{12}$. Em 2012, a pedido da própria escola, foi conduzido um amplo projeto para avaliar a saúde dos escolares, não apenas em questões nutricionais, mas também em relação à parâmetros bioquímicos e aparência dento-facial.

A amostra do estudo foi composta pelos adolescentes de 14 a 19 anos do ensino médio da escola, matriculados nos anos 2000 e 2012. Nos dois estudos foram excluídos da amostra aqueles alunos cujos pais ou eles próprios não concordaram com a participação no estudo e aqueles que, apesar de estarem matriculados, não frequentavam regularmente as aulas.

Em 2000, entre os meses de fevereiro a junho, uma equipe treinada de estudantes da Faculdade de Nutrição da Universidade Federal de Pelotas (UFPel) pesou e aferiu a altura dos adolescentes das seis escolas privadas analisadas. Os antropometristas foram submetidos a treinamento específico constituído de leitura e discussão de um roteiro com instruções para a coleta dos dados de peso e altura e posterior exercício prático de tomada de medidas e padronização, permitindo a comparação da precisão e exatidão das medidas e cálculo do erro técnico intra e interobservador. Os erros técnicos aceitáveis basearam-se na publicação de Habitch ${ }^{13}$ e a técnica para a tomada das medidas seguiu as recomendações de Lobman et al. ${ }^{14}$. Foi utilizada balança marca Filizola com precisão de $0,1 \mathrm{~kg}$ para aferição do peso e fita métrica inextensível presa na parede com precisão de $0,1 \mathrm{~cm}$ para verificação da altura. Os dados utilizados no presente estudo para fins de comparação referem-se a uma dessas seis escolas avaliadas, a qual foi novamente avaliada em 2012.

No inquérito de 2012, o qual ocorreu entre os meses de junho e agosto, a coleta de dados também foi realizada por estudantes da Faculdade de Nutrição/ UFPel submetidos ao mesmo treinamento e padronização para o trabalho de campo ocorridos no ano 2000. Para a aferição do peso corporal foi utilizada balança digital de bioimpedância marca Tanita Bc 558, com capacidade de $150 \mathrm{~kg}$ e precisão de $0,1 \mathrm{~kg}$. Para a medida da altura, foi utilizado um estadiômetro portátil e desmontável da marca Alturaexata, com precisão de $0,1 \mathrm{~cm}$ e altura máxima de 2,13 metros. Neste caso, a medida da altura foi tomada estando os jovens sem quaisquer adornos da cabeça (bonés, presilhas de cabelo e etc), descalços e em postura ereta com o olhar fixo no horizonte, conforme o plano de Frankfurt.

A avaliação da prática de atividade física foi realizada a partir de questionário desenvolvido e aplicado na mesma cidade em momento anterior. Os estudantes foram questionados de maneira autoaplicada quanto à frequência e duração em cada uma das dez diferentes atividades existentes no instrumento, além de haver a opção para inserirem as mesmas informações para outras atividades. Versão bastante similar deste instrumento mostrou-se repetível $(\mathrm{rho}=0,62)$ e válida $(\mathrm{kappa}=0,58)$ quando testado contra pedômetros ${ }^{15}$. O tempo semanal em cada atividade (frequência*duração) foi somado e foram considerados ativos os adolescentes que praticavam pelo menos $300 \mathrm{minu}-$ 
tos/semana de atividade física. Os adolescentes foram questionados também quanto ao hábito de assistir televisão, a partir de duas perguntas ainda não validadas, sendo considerado alto tempo diário igual ou superior a duas horas.

$\mathrm{O}$ estado nutricional dos adolescentes foi determinado, tanto no primeiro inquérito, quanto no segundo, a partir do índice de massa corporal (IMC) para idade em escore-z. O IMC é definido como a relação entre o peso $(\mathrm{kg})$ e o quadrado da altura $\left(\mathrm{m}^{2}\right)$. A proposta da Organização Mundial da Saúde para crianças e adolescentes de 5 a 19 anos $^{16}$ foi utilizada como referência, sendo consideradas as seguintes categorias e pontos de corte: escore-z inferior a -2 , magreza; escore-z entre -2 e +1 , eutrofia; escore-z superior a +1 , excesso de peso e superior a +2 , obesidade. As datas da avaliação antropométrica e do nascimento dos adolescentes foram utilizadas para obtenção da idade exata, no momento da tomada de medidas. A análise dos dados antropométricos foi conduzida no programa Anthro Plus.

A entrada de dados foi realizada com dupla digitação utilizando-se o programa Epidata versão 3.1. Foi realizada análise descritiva, apresentando-se as amostras conforme sexo e idade e também o diagrama de dispersão do escore-z do IMC para idade. As prevalências de excesso de peso e obesidade nos dois inquéritos foram associadas às variáveis independentes. A variação temporal do estado nutricional foi analisada utilizando-se a regressão de Poisson sendo mostrados valores de significância estatística a partir do teste da razão de verossimilhanças, enquanto que o teste do Qui-Quadrado de Pearson foi utilizado para avaliar a associação entre a prática de atividade física e tempo assistindo televisão e o excesso de peso e a obesidade no ano de 2012. A análise ajustada entre atividade física, tempo de televisão e excesso de peso e obesidade foi realizada a partir de regressão de Poisson com ajuste para variância robusta. As análises estatísticas foram conduzidas no software Stata 12.0. e foram consideradas estatisticamente significativas associações cujo $\mathrm{p}<0,05$.

O estudo foi aprovado pelo Comitê de Ética em Pesquisa da Faculdade de Medicina da Universidade Federal de Pelotas (protocolo 085/2011). Todos os pais ou responsáveis pelos alunos menores de 18 anos avaliados assinaram um termo de consentimento livre e esclarecido.

\section{RESULTADOS}

No primeiro inquérito, foram avaliados 479 dos 496 estudantes (3,4\% de perdas e recusas). Já em 2012, dos 540 estudantes, 529 foram entrevistados (98\% de resposta). Porém, foram medidos apenas 372 adolescentes $(31,1 \%$ de perdas e recusas). A idade média dos estudantes da escola entrevistados no ano 2000 foi de 15,3 anos $(d p=1,0)$ e no ano 2012 foi de 15,5 anos $(d p=1,0)$. Em 2012 foram entrevistadas 307 meninas $(58,0 \%)$ e 48,2\% dos jovens tinham idade igual ou superior a 16 anos. Os adolescentes com medidas antropométricas em ambos os anos de 2000 e 2012 não diferiram quanto ao sexo $(p=0,108)$ e idade ( $\mathrm{p}=0,343)$. A proporção de meninas com peso e altura avaliados em 2000 foi de $55 \%$, enquanto que em 2012 foi de $60 \%$.

A Tabela 1 mostra as associações transversais entre as variáveis demográficas avaliadas, atividade física e tempo de televisão e as prevalências de excesso de peso e obesidade dos inquéritos ocorridos em 2000 e 2012. Em ambos os inquéritos, as prevalências de excesso de peso e obesidade foram maiores para 
os meninos, porém no inquérito do ano 2000, associação significativa foi observada apenas quanto à obesidade, sendo que os meninos apresentaram prevalência de 5 pontos percentuais superior à observada entre as meninas. Em 2012, os meninos apresentaram excesso de peso significativamente maior que as meninas e não houve diferença significativa quanto à prevalência de obesidade. Tanto em 2000, quanto em 2012 não houve diferença nas prevalências de excesso de peso ou de obesidade por faixa etária.

TABELA 1 - Associação entre características dos adolescentes de escola privada de Pelotas (RS) e as prevalências de excesso de peso e obesidade nos anos de 2000 e 2012.

\begin{tabular}{|c|c|c|c|c|c|c|c|c|c|c|c|c|}
\hline \multirow{5}{*}{ Variáveis } & \multicolumn{12}{|c|}{ Ano de estudo } \\
\hline & \multicolumn{6}{|c|}{$2000(N=479)$} & \multicolumn{6}{|c|}{2012 ( $N=372)$} \\
\hline & \multicolumn{3}{|c|}{ Excesso de peso } & \multicolumn{3}{|c|}{ Obesidade } & \multicolumn{3}{|c|}{ Excesso de peso } & \multicolumn{3}{|c|}{ Obesidade } \\
\hline & \multicolumn{2}{|c|}{ Bruta } & \multirow{2}{*}{$\begin{array}{l}\text { Ajustada } \\
\left.\text { RP (IC }{ }_{95 \%}\right)\end{array}$} & \multicolumn{2}{|c|}{ Bruta } & \multirow{2}{*}{$\begin{array}{l}\text { Ajustada } \\
\text { RP }\left(\mid C_{95 \%}\right)\end{array}$} & \multicolumn{2}{|c|}{ Bruta } & \multirow{2}{*}{$\begin{array}{l}\text { Ajustada } \\
\text { RP }\left(I C_{95 \%}\right)\end{array}$} & \multicolumn{2}{|c|}{ Bruta } & \multirow{2}{*}{$\begin{array}{l}\text { Ajustada } \\
\text { RP }\left(I C_{95 \%}\right)\end{array}$} \\
\hline & $N(\%)$ & $\mathrm{p}$ & & $N(\%)$ & $\mathrm{p}$ & & $N(\%)$ & $\mathrm{p}$ & & $\mathrm{N}(\%)$ & $\mathrm{p}$ & \\
\hline Sexo & \multicolumn{3}{|c|}{0,286} & \multicolumn{3}{|c|}{0,009} & \multicolumn{3}{|c|}{0,004} & \multicolumn{3}{|c|}{0,176} \\
\hline Masculino & $51(23,7)$ & & $\begin{array}{c}1,19 \\
(0,85 ; 1,68)\end{array}$ & $17(7,9)$ & & $\begin{array}{c}2,91 \\
(1,23 ; 6,89)\end{array}$ & $58(39,5)$ & & $\begin{array}{c}1,59 \\
(1,18 ; 2,15)\end{array}$ & $18(12,2)$ & & $\begin{array}{c}1,54 \\
(0,83 ; 2,85)\end{array}$ \\
\hline Feminino & $52(19,7)$ & & 1,00 & $7(2,7)$ & & 1,00 & $56(24,9)$ & & 1,00 & $18(8,0)$ & & 1,00 \\
\hline Idade & \multicolumn{3}{|c|}{0,283} & \multicolumn{3}{|c|}{0,260} & \multicolumn{3}{|c|}{0,093} & \multicolumn{3}{|c|}{0,533} \\
\hline 14-15 anos & $62(23,3)$ & & 1,00 & $16(6,0)$ & & 1,00 & $52(26,8)$ & & 1,00 & $17(8,8)$ & & 1,00 \\
\hline$\geqslant 16$ anos & $41(19,3)$ & & $\begin{array}{c}0,83 \\
(0,59 ; 1,18)\end{array}$ & $8(3,8)$ & & $\begin{array}{c}0,66 \\
(0,29 ; 1,51)\end{array}$ & $62(34,8)$ & & $\begin{array}{c}1,31 \\
(0,97 ; 1,77)\end{array}$ & $19(10,7)$ & & $\begin{array}{c}1,23 \\
(0,66 ; 2,28)\end{array}$ \\
\hline $\begin{array}{l}\text { Atividade } \\
\text { física } \\
\text { ( } 2300 \mathrm{~min} / \\
\text { sem) }\end{array}$ & & & & & & & \multicolumn{3}{|c|}{0,206} & \multicolumn{3}{|c|}{0,174} \\
\hline Ativo & --- & & & --- & & & $44(34,9)$ & & 1,00 & $16(12,7)$ & & 1,00 \\
\hline Inativo & --- & & & --- & & & $69(28,5)$ & & $\begin{array}{c}0,88 \\
(0,65 ; 1,20)\end{array}$ & $20(8,3)$ & & $\begin{array}{c}0,69 \\
(0,37 ; 1,28)\end{array}$ \\
\hline $\begin{array}{l}\text { Tempo de } \\
\text { televisão } \\
\text { ( } \geqslant 2 \text { hs/dial }\end{array}$ & & & & & & & \multicolumn{3}{|c|}{0,009} & \multicolumn{3}{|c|}{0,193} \\
\hline Sim & --- & & & --- & & & $69(36,9)$ & & $\begin{array}{c}1,62 \\
(1,18 ; 2,22)\end{array}$ & $22(11,8)$ & & $\begin{array}{c}1,62 \\
(0,86 ; 3,06)\end{array}$ \\
\hline Não & --- & & & --- & & & $44(24,3)$ & & 1,00 & $14(7,7)$ & & 1,00 \\
\hline
\end{tabular}

Do total, 192 adolescentes em 2012 (36,6\%) atingiram a recomendação de pelo menos 300 minutos por semana de atividade física no lazer, enquanto que 50,3\% dos adolescentes assistiam televisão por no mínimo duas horas diárias. A prática de atividade física no lazer não foi associada com as prevalências de excesso de peso e obesidade dos estudantes entrevistados no ano 2012. Porém, tempo diário de televisão igual ou superior a duas horas foi associado com uma prevalência de excesso de peso de mais de 10 pontos percentuais em comparação com adolescentes que assistem menos de duas horas diárias de televisão $(\mathrm{p}=0,009)$. Essa associação permaneceu após ajuste para sexo e idade $(\mathrm{RP}=1,61 ; \mathrm{IC} 95 \%: 1,17 ; 2,21)$ e também para a prática de atividade física $(\mathrm{RP}=1,62 ; \mathrm{IC} 95 \%: 1,12 ; 2,22)$.

A Figura 1 mostra as atividades praticadas no período de lazer pelos adolescentes entrevistados no ano de 2012. O futebol e a musculação foram as atividades mais praticadas pelos meninos, enquanto que a musculação e a caminhada foram as atividades mais praticadas no lazer pelas meninas.

Com relação à variação temporal nas prevalências de excesso de peso e obesidade entre 2000 e 2012, pode-se observar na Figura 2 que houve um 
grande incremento na prevalência de excesso de peso nos estudantes desta escola (43\%, p=0,009), passando de 21,5\% em 2000, para 30,7\% em 2012. Para a obesidade, a taxa de ganho foi ainda maior $(93 \%, \mathrm{p}=0,01)$, já que a prevalência praticamente dobrou de um ano para o outro, passando de 5,0\% em 2000 para $9,7 \%$ em 2012. Ajustando-se a mudança na prevalência de ambos os desfechos para as informações de sexo e idade dos adolescentes, as taxas de aumento nas prevalências de excesso de peso e obesidade foram de $45 \%(\mathrm{p}=0,005)$ e $101 \%$ $(\mathrm{p}=0,004)$, respectivamente. Ainda, de acordo com a Figura 3, observa-se que no período de 12 anos ocorreu um desvio para a direita na curva de escore-z de IMC para idade. As médias e medianas de escore- $z$ variaram respectivamente de 0,26 e 0,19, no ano 2000, para 0,5 e 0,4, no ano de 2012 .

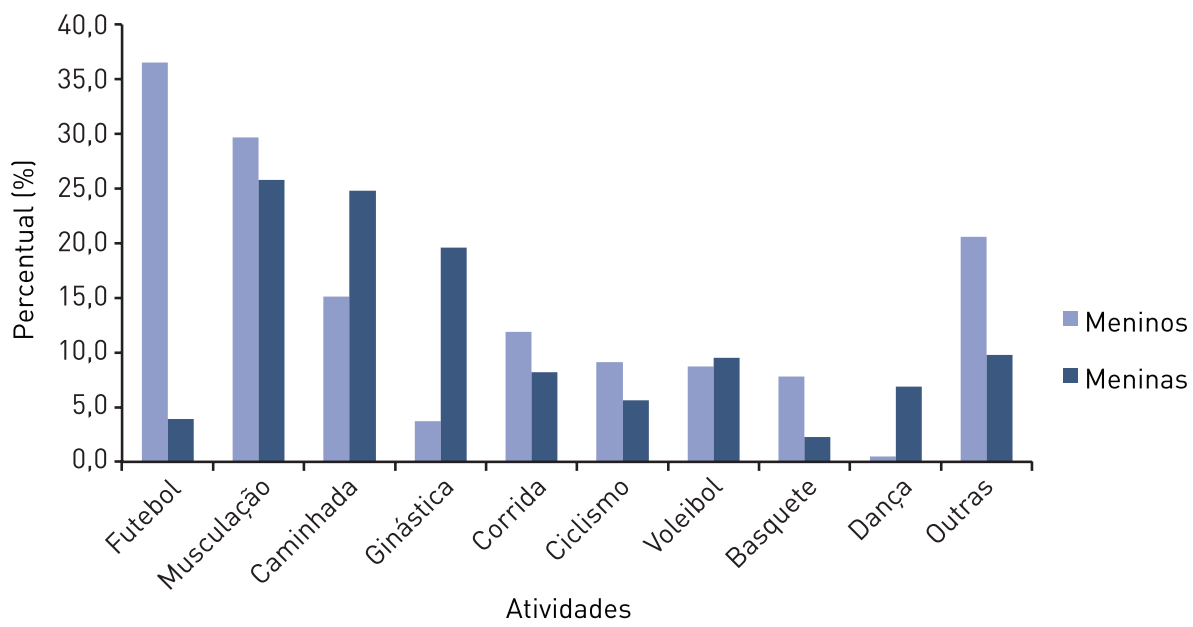

FIGURA 1 - Atividades físicas praticadas durante o período de lazer por alunos do ensino médio de escola privada de Pelotas, RS. 2012.

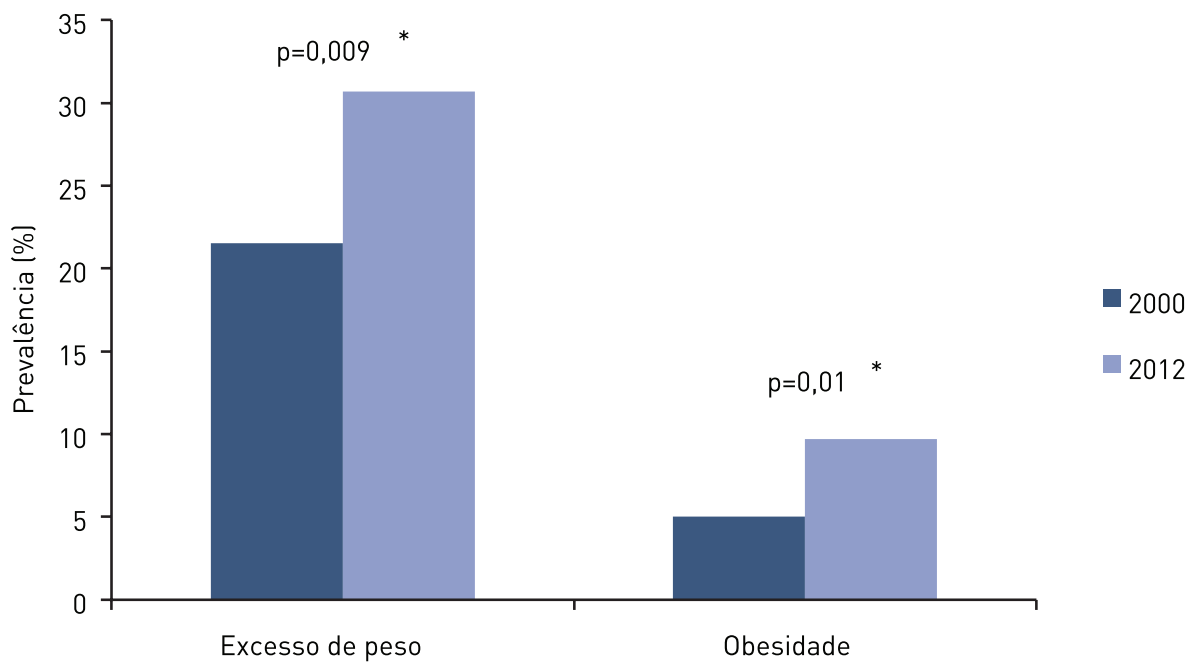

FIGURA 2 - Variação temporal na prevalência de excesso de peso e obesidade de adolescentes de escola privada de Pelotas (RS) entre os anos 2000 e 2012.

* Valor-p referente ao teste da Razão de Verossimilhanças. 

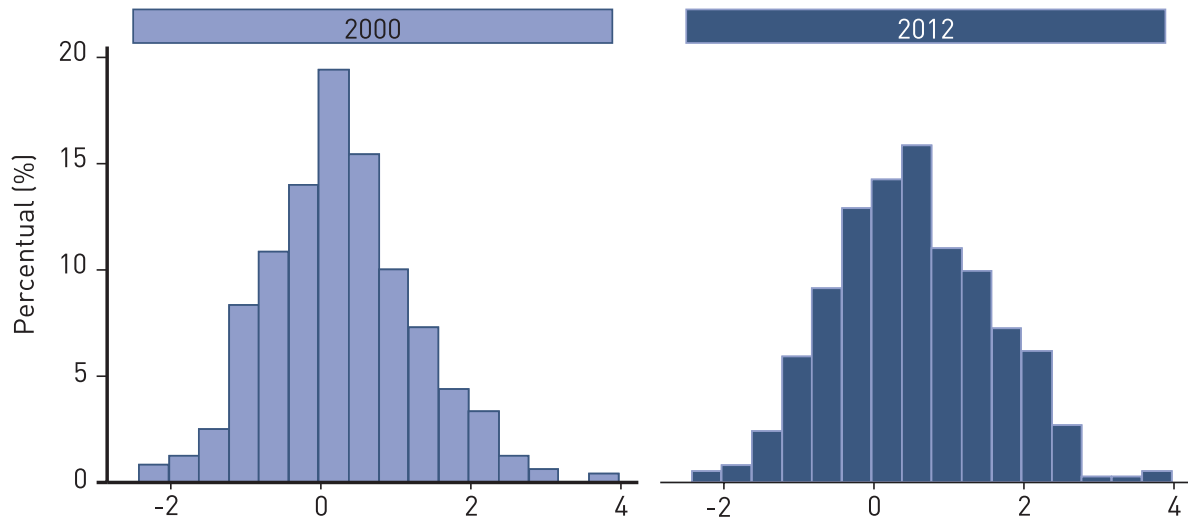

Graphs by survey

Escore-z IMC por idade

FIGURA 3 - Histograma da distribuição do escore-z do IMC por idade nos inquéritos de 2000 e de 2012 para os adolescentes de escola privada de Pelotas (RS).

A Figura 4 demonstra a mudança ocorrida nas prevalências de excesso de peso e obesidade de acordo com o sexo e faixa etária dos adolescentes, respectivamente, nos dois inquéritos. Entre 2000 e 2012, observou-se um aumento estatisticamente significativo na prevalência de excesso de peso no sexo masculino, a qual variou de 23,7\% em 2000, para 39,5\% em 2012 (p=0,008). Em contraparti$\mathrm{da}$, quanto à obesidade, $\mathrm{o}$ aumento foi significativo apenas para as meninas, sendo que em 2000 a prevalência encontrada foi de 2,7\% e em 2012, 8,0\% ( $\mathrm{p}=0,009)$. Quando observada a variação nas prevalências de excesso de peso e obesidade em relação às idades dos adolescentes, os maiores incrementos se deram entre os mais velhos, enquanto que incrementos observados entre os adolescentes de 14 e 15 anos não foram estatisticamente significativos. Assim, naqueles que estavam com 16 anos ou mais nos dois inquéritos, a prevalência de excesso de peso de $19,3 \%$ em 2000 foi para 34,8\% em 2012 ( $\mathrm{p}=0,003)$, enquanto que a prevalência de obesidade passou de 3,8\% em 2000, para 10,7\% em 2012 ( $\mathrm{p}=0,009)$.

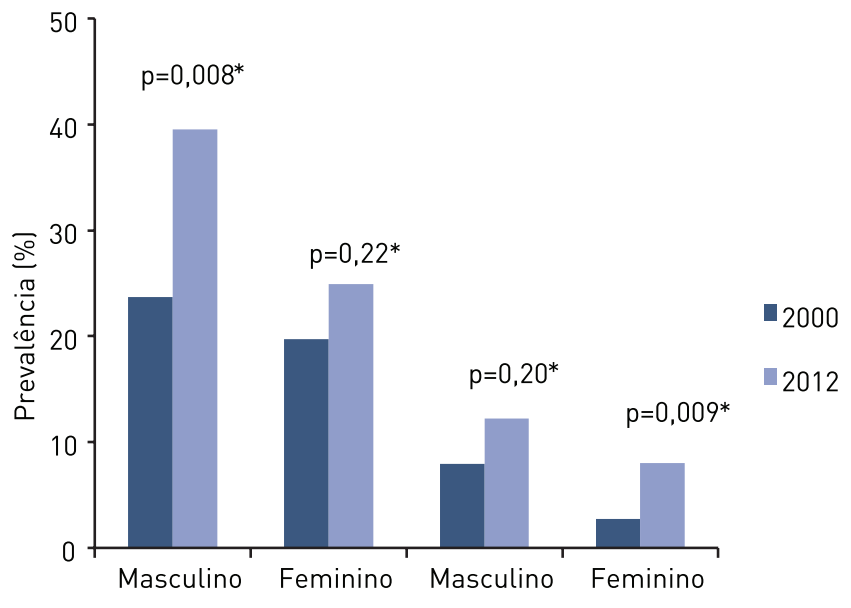

Excesso de peso

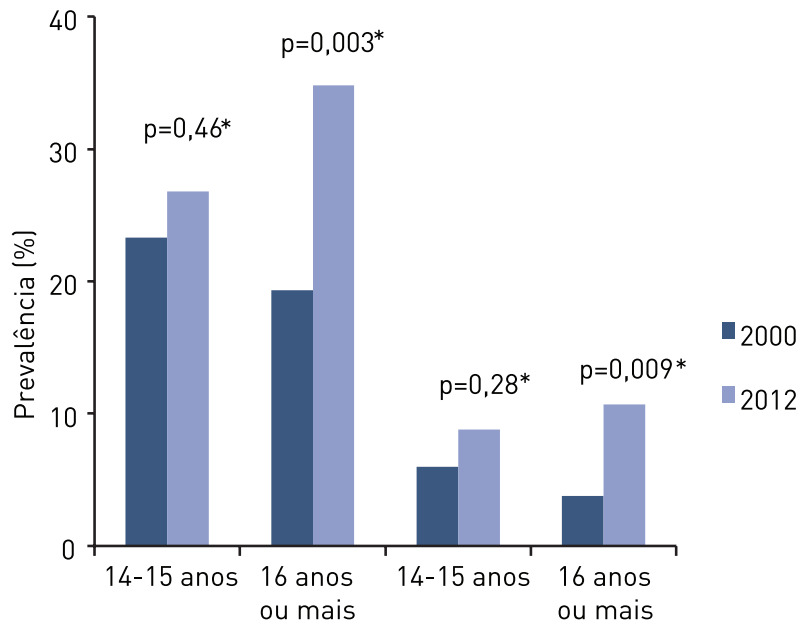

Excesso de peso

Obesidade

FIGURA 4 -Variação temporal nas prevalências de excesso de peso e obesidade conforme sexo e idade dos adolescentes de escola privada de Pelotas (RS) entre os anos 2000 e 2012.

* Valor-p referente ao teste da Razão de Verossimilhanças. 


\section{DISCUSSÃO}

Diante dos resultados apresentados, observa-se que o excesso de peso aumentou consideravelmente nesses doze anos e a taxa de obesidade aumentou em quase $100 \%$. Em ambos os inquéritos, maiores prevalências dos desfechos foram encontradas no sexo masculino. Porém, ao avaliar a mudança ocorrida de um período para o outro, observa-se que o aumento para a obesidade foi maior entre as meninas, indicando que o problema atinge ambos os sexos. Em relação à idade, aumento significativo foi observado somente para os adolescentes mais velhos. O presente estudo não encontrou associação entre as prevalências de excesso e obesidade e prática de atividade física no lazer nos adolescentes entrevistados e medidos em 2012. Porém, o tempo assistindo televisão foi associado negativamente com a prevalência de excesso de peso, independente de possíveis confundidores e da própria prática de atividade física.

Considerando-se que neste estudo foram diagnosticados com excesso de peso adolescentes que apresentaram IMC para idade acima de 1 desvio padrão em relação à mediana esperada para a idade, o valor de referência de acordo com uma distribuição normal seria de aproximadamente 16,0\% de excesso de peso. Assim, observa-se que em 2000 a prevalência excesso de peso de 21,5\% foi aproximadamente $30,0 \%$ acima desse valor esperado, enquanto que em 2012 a prevalência encontrada de $30,7 \%$ esteve aproximadamente $90 \%$ acima do valor de referência, ou seja, quase o dobro. Isso reflete a ausência de intervenções planejadas e executadas no mesmo período voltadas direta ou indiretamente à prevenção ou tratamento do excesso de peso e obesidade.

A cidade de Pelotas possui programas de atividades de lazer bastante pontuais e muito pouco voltados à população jovem. Além disso, políticas públicas voltadas à melhoria da alimentação escolar não são de alcance à população deste estudo, sendo que inexiste lei de controle à venda de determinados alimentos nas cantinas. Cabe ainda salientar que a cidade não possui Núcleos de Apoio à Saúde da Família (NASFs), de modo que podemos afirmar que a população adolescente da cidade não esteve suscetível a nenhuma intervenção para redução da ocorrência de excesso de peso e obesidade, o que é preocupante não apenas ao subgrupo estudado nesta análise através da amostra em questão, mas aos adolescentes da cidade como um todo.

Comparações entre os resultados encontrados neste estudo com outras publicações devem ser feitas com cautela, uma vez que diferentes critérios ainda são utilizados para classificar o estado nutricional de adolescentes ${ }^{5}$. Além disso, estudos que avaliaram mudança ou tendência temporal nas prevalências de excesso de peso e obesidade em populações específicas de adolescentes brasileiros são pouco referidos na literatura.

Em relação aos estudos nacionalmente representativos, pode-se observar que a prevalência desses desfechos mais que duplicou entre os anos 19741975 e $1989^{6}$. Especificamente para a região Sul, entre os anos 2002-2003 e 2008-2009, a prevalência de excesso de peso nos meninos passou de $21,0 \%$ para $27,2 \%$ e a obesidade, de $6,1 \%$ para $7,7 \%$. No sexo feminino, o excesso de peso aumentou de $16,6 \%$ para $22,0 \%$, enquanto que a obesidade passou de $3,3 \%$ para $5,4 \%$, sendo a região onde foram identificadas as maiores prevalências no país ${ }^{6}$. Assim como nos resultados encontrados nesse estudo, a prevalência de excesso de peso nos adolescentes participantes da Pesquisa de 
Orçamentos Familiares (POF 2008-2009) foi maior nos meninos ${ }^{6}$. Na POF também foram encontradas maiores prevalências de excesso de peso naqueles adolescentes pertencentes às famílias de maior renda ${ }^{6}$, a qual representa uma parcela da população mais semelhante à deste estudo. Com relação aos resultados da PeNSE, tendo como referência apenas os estudantes de escola privada, a prevalência de excesso de peso de $31,0 \%{ }^{4}$ nestes adolescentes foi bastante similar ao apresentado no presente estudo (30,7\%).

Resguardando-se as diferenças metodológicas de avaliação, em inquéritos domiciliares realizados em Pelotas não foram encontradas diferenças significativas nas prevalências de excesso de peso e obesidade entre os $\operatorname{sexos}^{17}$. Em contrapartida, estudo realizado em Recife $(\mathrm{PE})^{18}$, o qual incluiu apenas adolescentes provenientes da rede privada de ensino, identificou as maiores prevalências nos meninos, assim como neste estudo.

Com relação à idade, inquéritos conduzidos em Pelotas não encontraram associação com as prevalências de excesso de peso e obesidade ${ }^{19,17}$. No entanto, Guedes et al..$^{20}$, em estudo com crianças e adolescentes escolares do Paraná, observaram que o aumento do sobrepeso e obesidade ocorreu conforme o aumento da idade, sobretudo entre os meninos, sendo que a faixa etária dos 15 aos 18 anos foi a que apresentou as maiores prevalências.

Evidências da literatura demonstram que mesmo que a ascensão do excesso de peso e da obesidade ocorra de forma distinta de acordo com o grau de desenvolvimento de cada país, tanto nos países desenvolvidos quanto nos países em desenvolvimento, a tendência de aumento nas prevalências é ainda visível $^{21}$. Estudos realizados com crianças e adolescentes nos Estados Unidos e Europa demonstraram que a prevalência de obesidade para adolescentes triplicou nos últimos 30 anos, e aumentos relevantes também foram observados na Ásia, Oceania e América Latina ${ }^{21}$. Contudo, uma diferença que pode ser destacada é que nos países desenvolvidos as maiores prevalências e os maiores aumentos têm sido constatados em crianças e adolescentes de menor nível socioeconômico, como foi demonstrado em estudos realizados na Austrália ${ }^{22}$ e Estados Unidos ${ }^{23}$. Em contrapartida, nos países em desenvolvimento, como Índia ${ }^{24}$, México $^{25}$ e Brasil $^{6}$ as maiores prevalências e os maiores incrementos no decorrer do tempo, estão mais ligados ao maior nível socioeconômico. Tal contraste talvez possa ser explicado pelos diferentes estágios da transição nutricional que esses países enfrentam, conforme descritos por Popkin ${ }^{26}$.

Além de hábitos alimentares inadequados ${ }^{27}$, o aumento do excesso de peso ao longo do tempo parece ocorrer paralelamente à diminuição na prática de atividade física em adolescentes ${ }^{10}$. Neste estudo não encontrou-se associação entre prática de atividade física no lazer e prevalências de excesso de peso e obesidade. Porém, tempo superior a duas horas assistindo televisão foi associado ao excesso de peso. A ausência de associação com a prática de atividade física pode se dar em virtude do viés de causalidade reversa pois, uma vez que estado nutricional e atividade física foram avaliados em um mesmo momento, pode-se supor que a ocorrência dos desfechos (excesso de peso e/ou obesidade) possa ter levado à prática de atividade física durante o lazer por estes adolescentes, o que não aconteceu com relação ao tempo assistindo televisão por ser um comportamento talvez mais difícil de ser modificado.

A prevalência de prática de atividade física nesse estudo foi de aproximadamente 37,0\%. Em estudo transversal realizado em Pelotas, Dumith e co- 
laboradores ${ }^{28}$ encontraram prevalência de atividade física total de 48,2\% em adolescentes de 14-15 anos, ou seja, menos da metade dos jovens foi considerada fisicamente ativa (pelo menos 300 minutos/semana de atividade física moderada ou vigorosa). $\mathrm{O}$ mesmo estudo constatou que essa proporção tende a diminuir com o aumento do nível econômico, o que corrobora com a menor prevalência encontrada nesta investigação e, ainda, que as meninas foram menos ativas que os meninos, assim como ocorreu na presente amostra (dados não apresentados).

Como limitação deste estudo deve-se destacar o alto percentual de perdas e recusas para as medidas antropométricas do segundo inquérito $(31,1 \%)$, as quais foram similares quanto ao sexo $(52,9 \%$ no sexo feminino e $47,1 \%$ no sexo masculino), mas concentradas nos adolescentes do primeiro ano do ensino médio $(43,5 \%)$. Convém ressaltar que muitos adolescentes se negaram a participar da pesquisa, justamente por motivo de constrangimento em relação a expor a medida de seu peso corporal para terceiros, podendo-se supor que, provavelmente, se houvesse menos perdas e recusas, as prevalências de excesso de peso e obesidade encontradas poderiam ter sido ainda maiores. Ainda, a utilização de diferentes instrumentos para aferição da estatura dos adolescentes pode ser considerada outra limitação. Entretanto, considerandose a mesma técnica na tomada das medidas e também a mesma precisão dos instrumentos acredita-se que esta diferença não tenha afetado os resultados.

Ressalta-se como aspecto positivo desse estudo o fato de se ter disponível um diagnóstico da mudança observada ao longo do tempo nas prevalências de excesso de peso e obesidade nesta população. Os achados da presente investigação poderão servir como linha de base para futuros monitoramentos de tendência, assim como fonte de comparação para outros estudos com adolescentes escolares brasileiros de origem similar, pois, embora oriundos de uma única escola da cidade, não há razões para supor que os adolescentes estudantes desta escola participante do estudo sejam diferentes de alunos de outras escolas particulares da cidade. Ainda, estudos descritivos que analisam variação ou tendência temporal podem ser significativos para a busca dos fatores que influenciaram a mudança ${ }^{29}$, bem como investigar possíveis alterações nas condições de vida que possam estar relacionadas com modificações observadas ao longo do tempo.

A escola indubitavelmente se constitui como um ambiente propício para a realização de ações que promovam a prevenção e o controle do avanço dos problemas nutricionais. Porém, as elevadas prevalências encontradas nesse estudo podem indicar que o acesso a estratégias e intervenções de promoção à prática de atividades físicas, redução do comportamento sedentário e incentivo à alimentação saudável que visem a prevenção desses fatores, provavelmente não estão sendo eficientes ou inexistem nesse grupo populacional.

\section{Contribuições dos autores}

MOX participou da concepção do estudo, revisão de literatura, coleta dos dados e redação do manuscrito; RMB participou da concepção do estudo, análise dos dados e redação do manuscrito. FVM coordenou a coleta de dados e participou na revisão do manuscrito. MBN participou da coleta de dados e da revisão do manuscrito. DPG participou da concepção do estudo, análise dos dados e revisão do manuscrito. 


\section{REFERÊNCIAS}

1. Popkin BM. The nutrition transition and its health implications in lower-income countries. Public Health Nutr. 1998 Mar; 1(1):5-21.

2. Batista Filho M, Rissin A. A transição nutricional no Brasil: tendências regionais e temporais. Cad Saude Publica. 2003; 19 Suppl 1:S181-91.

3. Suáres A, Argüilles JM. Nutritional evaluation of adolescents: usefulness of anthropometric indicators in the diagnosis of obesity. Acta Paediatr. Hungarica. 1986; 27(4):303-10.

4. Araújo C, Toral N, Silva AC, Velasquez-Melendez G, Dias AJ. Estado nutricional dos adolescentes e sua relação com variáveis sociodemográficas: Pesquisa Nacional de Saúde do Escolar (PeNSE), 2009. Cien Saude Colet. 2010 Oct; 15 Suppl 2:3077-84.

5. Tassitano RM, Tenório MCM, Hallal PC. Revisão sistemática sobre obesidade em adolescentes brasileiros. Rev Bras Cineantropom Desempenho Hum. 2009; 11(4):449-456.

6. Instituto Brasileiro de Geografia e Estatística - IBGE. Pesquisa de orçamentos familiares 2008-2009: antropometria e estado nutricional de crianças, adolescentes e adultos no Brasil. Rio de Janeiro: IBGE; 2010.

7. Enes CC, Slater B. Obesidade na adolescência e seus principais fatores determinantes. Rev Bras Epidemiol. 2010 Mar; 13(1):163-71.

8. Tailor AM, Peeters PH, Norat T, Vineis P, Romaguera D. An update on the prevalence of the metabolic syndrome in children and adolescents. Int J Pediatr Obes. 2010 May 3; 5(3):202-13.

9. Hallal PC, Knuth AG, Cruz DK, Mendes MI, Malta DC. Prática de atividade física em adolescentes brasileiros. Cien Saude Colet. 2010 Oct; 15 Suppl 2:3035-42.

10. Knuth AG, Hallal PC. Temporal trends in physical activity: a systematic review. J Phys Act Health. 2009 Sep; 6(5):548-59.

11. Coll CVN, Knuth AG, Bastos JP, Hallal PC, Bertoldi AD. Time Trends of Physical Activity Among Brazilian Adolescents Over a 7-Year Period. Journal of Adolescent Health 2013; 1-5.

12. Neutzling MB. Fatores de risco para sobrepeso/obesidade em escolares adolescentes de Pelotas, RS, Brasil: um estudo de caso-controle [tese doutorado]. São Paulo: Escola Paulista de Medicina da Universidade Federal de São Paulo; 2002.

13. Habitcht JP. Estandarizacion de metodos epidemiológicos cuantitativos sobre el terreno. Bol Oficina Saint Panam. 1974 Mayo; 76(5):375-84.

14. Lohman TG, Roche AF, Martorell R, editors. Anthropometric Standardization Reference Manual. Abridged ed Champaign, IL: Human Kinetics Books; 1988.

15. Bastos, JP, Araujo CL, Hallal PC. "Prevalence of insufficient physical activity and associated factors in Brazilian adolescents.”J Phys Act Health. 2008; 5(6):777-94.

16. De Onis M, Onyango AW, Borgui E, Siyam A, Nishida C, Siekmann J. Development of a WHO growth reference for school-aged children and adolescents. Bull World Helath Organ. 2007 Sep; 85(9):660-7.

17. Terres NG, Pinheiro RT, Horta BL, Pinheiro KA, Horta LL. Prevalência e fatores associados ao sobrepeso e à obesidade em adolescentes. Rev Saude Publica 2006 Aug; 40(4):627-33.

18. Balaban G, Silva GAP. Prevalência de sobrepeso e obesidade em crianças e adolescentes de uma escola da rede privada de Recife.J Pediatr (Rio J). 2001 Mar-Apr; 77(2):96-100.

19. Dutra CL, Araujo CL, Bertoldi AD. Prevalência de sobrepeso em adolescentes: um estudo de base populacional em uma cidade no Sul do Brasil. Cad Saude Publica. 2006 Jan; 22(1):151-62.

20. Guedes DP, Paula IG, Guedes JERP, Stanganelli LCR. Prevalência de sobrepeso e obesidade em crianças e adolescentes: estimativas relacionada ao sexo, à idade e à classe socioeconômica. Rev bras Educ Fís Esp, São Paulo. 2006 Jun-Set; 20(3):151-63.

21. Wang Y, Lobstein T. Worldwide trends in childhood overweight and obesity. Int J Pediatr Obes. 2006; 1(1):11-25.

22. O'Dea JA, Dibley MJ. Obesity increase among low SES Australian schoolchildren between 2000 and 2006: time for preventive interventions to target children from low imcome schools? Int J Public Health. 2010 Jun; 55(3):185-92. 
23. Ogden CL, Lamb MM, Carroll MD, Flegal KM. Obesity and socioeconomic status in children and adolescents: United States, 2005-2008. NCHS Data Brief. 2010 Dec; (51):1-8.

24. Gupta DK, Shah P, Misra, A, Bharadwaj S, Gulati S, Gupta N, et al. Secular trends in prevalence of overweight and obesity from 2006 to 2009 in urban asian Indian adolescents aged 14-17 years. PLoS One. 2011; 6(2):e17221.

25. Bonvecchio A, Safdie M, Monterrubio EA, Gust T, Villalpando S, Rivera JA. Overweight and obesity trends in Mexican children 2 to 18 years of age from 1988 to 2006. Salud Publica Mex. 2009; 51 Suppl 4:S586-94.

26. Popkin B. The dynamics of the dietary transition in the developing world. In: Caballero B, Popkin B, eds. The nutrition transition: diet and disease in the developing world. London: Academic Press, 2002: 111-28.

27. Levy RB, Castro IR, Cardoso Lde O, Tavares LF. Sardinha LM, Gomes Fda S, et al. Consumo e comportamento alimentar entre adolescentes brasileiros: Pesquisa Nacional de Saúde do Escolar (PeNSE), 2009. Cien Saude Colet. 2010 Oct; 15 Suppl 2:3085-97.

28. Dumith SC, Domingues MR, Gigante DP, Hallal PC, Menezes AM, Kohl HW. Prevalence and correlates of physical activity among adolescents from Southern Brazil. Rev Saude Publica. 2010 Jun; 44(3):457-67.

29. Latorre MRDO, Cardoso MRA. Análise de séries temporais em epidemiologia: uma introdução sobre os aspectos metodológicos. Rev Bras Epidemiol. 2001; 4(3):145-52.

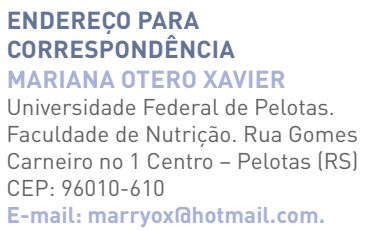

